
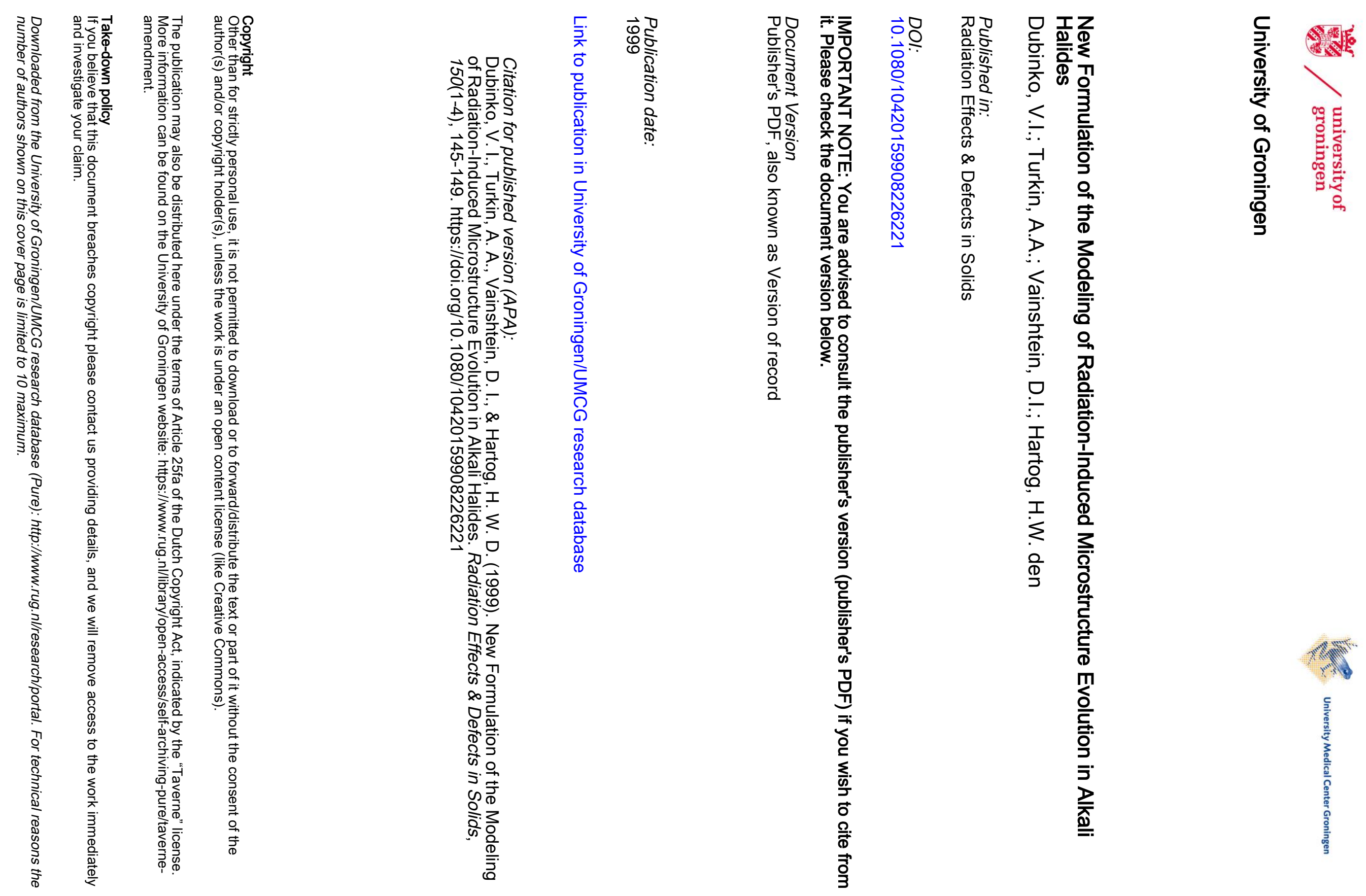


\title{
NEW FORMULATION OF THE MODELING OF RADIATION-INDUCED MICROSTRUCTURE EVOLUTION IN ALKALI HALIDES
}

\author{
V.I. DUBINKO ${ }^{a}$, A.A. TURKIN ${ }^{a}$, D.I. VAINSHTEIN ${ }^{\text {b,* }}$ \\ and H.W. DEN HARTOG ${ }^{\mathrm{b}}$ \\ ${ }^{a}$ Kharkov Institute of Physics and Technology, 310108 Kharkov, Ukraine; \\ ${ }^{\mathrm{b}}$ Solid State Physics Laboratory, University of Groningen 4 Nijenborgh, \\ NL-9747 AG Groningen. The Netherlands
}

(Received 6 July 1998; In final form 20 September 1998)

\begin{abstract}
Recent experimental results on heavily irradiated natural and synthetic $\mathrm{NaCl}$ crystals give a strong evidence for the formation of large vacancy voids, which cannot be explained by the Jain-Lidiard model used up to date for description of metal colloids and dislocation loops formed during earlier stages of irradiation. We propose a new mechanism of dislocation climb, which involves production of cation vacancies (with a trapped hole) as a result of absorption of extra $H$ centers. We consider an additional set of reactions between experimentally observed extended defects (dislocations, metal colloids, gas bubbles and voids) and point defects. The latter include $F$ and $H$ centers that are the primary defects produced by irradiation, and cation vacancies that are secondary defects produced in the process of dislocation climb. We show that highly overpressurized bubbles of fluid halogen are strongly biased for absorption of $H$ centers, which makes them grow via punching out interstitial dislocation loops. The loops grow and produce cation vacancies that are subsequently trapped at the surfaces of extended defects, such as the incoherent colloids, and recombine with extra $F$ centers giving rise to the void formation. Elastic interaction between extended defects and point defects is shown to play a major role, since it determines the bias factors of extended defects, which is a major driving force of the microstructural evolution under irradiation.
\end{abstract}

Keywords: Radiation damage; Alkali halides; Microstructure evolution

* Corresponding author. 


\section{INTRODUCTION}

It had been established as early as in the 1970 s that, at temperatures above room temperature, the principal radiation damage in alkali halides consists of clusters of halogen molecules and of complementary inclusions of alkali metal ("colloids") formed by agglomerating $F$ centers [1]. There was also evidence at that time that clusters of halogen molecules were in the form of bubbles of fluid halogen. So the question was [1] "how does the system of primary defects - interstitial $H$ centers and vacancy $F$ centers - separates out into bubbles and metal colloids?" Hobbs et al. [2] showed that irradiation at low temperatures (where vacancy defects were quite immobile) led to the formation of perfect interstitial dislocation loops. Such perfect loops require both interstitial halogen and interstitial alkali metal: but all previous work showed that ionization damages only the halide sub-lattice. The accepted explanation was that a cluster of some small number of halogen molecules formed an interstitial platelet, i.e. a faulted loop, which then at a certain size "unfaulted" to give the observed perfect loop structures and a corresponding number of dispersed halogen molecular centers [1]. Then Jam and Lidiard [3] have formulated a model, according to which, the dislocation bias for $H$ centers was the driving force for the colloid growth in alkali halides as it was for the void growth in metals under irradiation. However, the model left the dispersed molecular centers out of account merely as "debris" formed as a result of the $H$ aggregation and associated dislocation climb processes [1]. Their inclusion in the rate equations as the $F$ recombination sites would have the effect of so reducing the steady $F$ concentration that colloid growth would be impossible [1]. In spite of this and some other apparent problems, the Jain-Lidiard model was and still is considered to work well providing a qualitative explanation of the radiation damage in alkali halides.

From our results [4-6] obtained for $\mathrm{NaCl}$ irradiated up to $150 \mathrm{Grad}$ (which is about 30 displacements per atom), it follows that large vacancy voids (up to hundreds of $\mathrm{nm}$ in size) form in $\mathrm{NaCl}$ samples (with particular dopants), which cannot be explained by the conventional theory. In order to explain these results we present a new model of radiation damage in alkali halides. 


\section{NEW INSIGHT IN THE MICROSTRUCTURE EVOLUTION IN ALKALI HALIDES}

We will again use an analogy with radiation damage in metals. The elastic interaction between inclusions or cavities (which can be voids, gas bubbles or solid precipitates) and point defects (PD) was shown to result in the inclusion bias for absorption of $\mathrm{PD}$ of a certain kind depending on the inclusion size and the normal stress at its boundary [7]. The difference between the bias factors of extended defects (ED) of different kinds or sizes is the main driving force of the microstructural evolution under irradiation.

\subsection{The Role of Halogen Bubbles}

Based upon the above mentioned results [7], we can write the following expression for the bias of a cavity of a radius $R$ for the absorption of interstitial point defects, i.e. $H$ centers in the case under consideration:

$$
\delta_{\mathrm{c}}\left(R, \sigma_{\mathrm{rr}}\right)=\frac{\alpha_{\mathrm{im}} b}{R}+\alpha_{\mu}\left(\frac{\sigma_{\mathrm{rr}}}{\mu}\right)^{2},
$$

where the dimensionless constants $\alpha_{\mathrm{im}}$ and $\alpha_{\mu}$ are the material constants [6] corresponding to the "image" and "modulus" elastic interaction between the inclusion and PD, respectively, $b$ is the interatomic spacing, $\mu$ is the shear modulus of the matrix and $\sigma_{\mathrm{rr}}$ is the normal stress at the inclusion boundary, which, for a gas-filled cavity, is given by the difference between the surface tension, $2 \gamma / R$, and the gas pressure, $P$ : $\sigma_{\mathrm{rr}}=2 \gamma / R-P$, where $\gamma$ is the surface energy. Now, it follows from Eq. (1) that highly overpressurized $(P \gg 2 \gamma / R)$ halogen bubbles can be strongly biased for $H$ absorption, which can be a primary driving force for the separation of the $H$ and $F$ centers into bubbles and metal colloids. When several $H$ centers come together they combine to form a halogen bubble which "digs its own hole" in the lattice by punching out a perfect self-interstitial loop (SIA-loop). This process is exactly analogous to the loop-punching from helium bubbles in metals that was predicted as early as in 1959 [8] and confirmed experimentally ever since $[9,10]$. It is more favorable energetically than the production of a 
number of dispersed molecule centers as it was assumed in the Jain-Lidiard model. The threshold pressure for the loop-punching is inversely proportional to the bubble radius: $\sigma_{\mathrm{rr}}^{\text {th }} \approx-(\mu b+2 \gamma) / R$. It means that small enough halogen bubbles have a higher bias for $H$ centers than that of dislocations, and they can transform diffusion influxes of radiation produced $H$ centers into perfect SIA-loops. Both of these defects can be observed experimentally.

\subsection{Vacancy Void Formation}

When an $H$ center approaches dislocation, it forms a stoichiometric di-interstitial (needed for the dislocation climb) leaving behind a hole trapped in a cation vacancy ( $C V$ ) that is even more mobile than the $F$ center. Coherent colloids are transparent for $\mathrm{CV}$, but as soon as the colloids lose coherency, they start to trap $\mathrm{CV}$ that would react with incoming $F$ centers to produce stoichiometric divacancies that would subsequently convert incoherent colloids into vacancy voids attached to colloids. Voids grow faster than colloids, since they have no misfit stress and so have smaller bias to $H$ centers, $\delta_{\mathrm{V}}$, as compared to that of coherent colloids, $\delta_{\mathrm{C}}$ :

$$
\begin{gathered}
\delta_{\mathrm{V}}=\alpha_{\mathrm{im}} b / R_{\mathrm{V}}, \quad \delta_{\mathrm{C}}=\alpha_{\mathrm{im}} b / R_{\mathrm{C}}+\alpha_{\mu}\left(\sigma_{\varepsilon} / \mu\right)^{2}, \\
\sigma_{\varepsilon}=8 \mu_{\mathrm{C}} \varepsilon /\left(1+2 \mu_{\mathrm{C}} / \mu\right),
\end{gathered}
$$

where $\mu_{\mathrm{C}}$ is the colloid shear modulus, and $\varepsilon$ is the misfit that determines also a threshold colloid radius for the loss of coherency $R_{\mathrm{C}}^{\mathrm{th}} \approx \mu b / \sigma_{\varepsilon}$.

A quantitative comparison of the model predictions with experimental data is presented in the companion paper [6], which shows that voids can grow to the dimensions exceeding even the mean distance between colloids and bubbles, eventually absorbing them, and, hence, bringing the halogen gas and metal to a back reaction [5]. Thus, the present model offers a natural explanation not only for the void formation, but also for the observed release of stored energy with increasing dose of irradiation.

\section{Acknowledgements}

This study is supported by the Dutch Ministry for Economic Affairs. 


\section{References}

[1] A.B. Lidiard, Comments Solid State Phys. 8, 73 (1978).

[2] L.W. Hobbs et al., Proc. Roy. Soc. (Lond.) A332, 167 (1973).

[3] Uma Jain and A.B. Lidiard, Phil. Mag. 35, 245 (1977).

[4] D.I. Vainshtein, C. Altena and H.W. Den Hartog, Mater. Sci. Forum. 239-241. 607 (1997).

[5] H.W. Den Hartog and D.I. Vainshtcin, Mater. Sci. Forum. 239-241, 611 (1997).

[6] D.I. Vainshtein, V.I. Dubinko, A.A. Turkin and H.W. den Hartog, Void formation in heavily irradiated $\mathrm{NaCl}$, These proceedings.

[7] V.I. Dubinko, J. Nucl. Mater. 206, 1 (1993).

[8] G.W. Greenwood, A.J.E. Foreman and D.E. Rimmer, J. Nucl. Mater. 4, 305 (1959).

[9] J.H. Evans, A. Van Veen and L.M. Caspers, Radiat. Eff. 78, 105 (1983).

[10] V.I. Dubinko, V.V. Slezov, A.V. Tur and V.V. Yanovskij, Radiat. Eff. 100, 85 (1986). 